\title{
Medical Image of the Week: Splenic Infarction
}

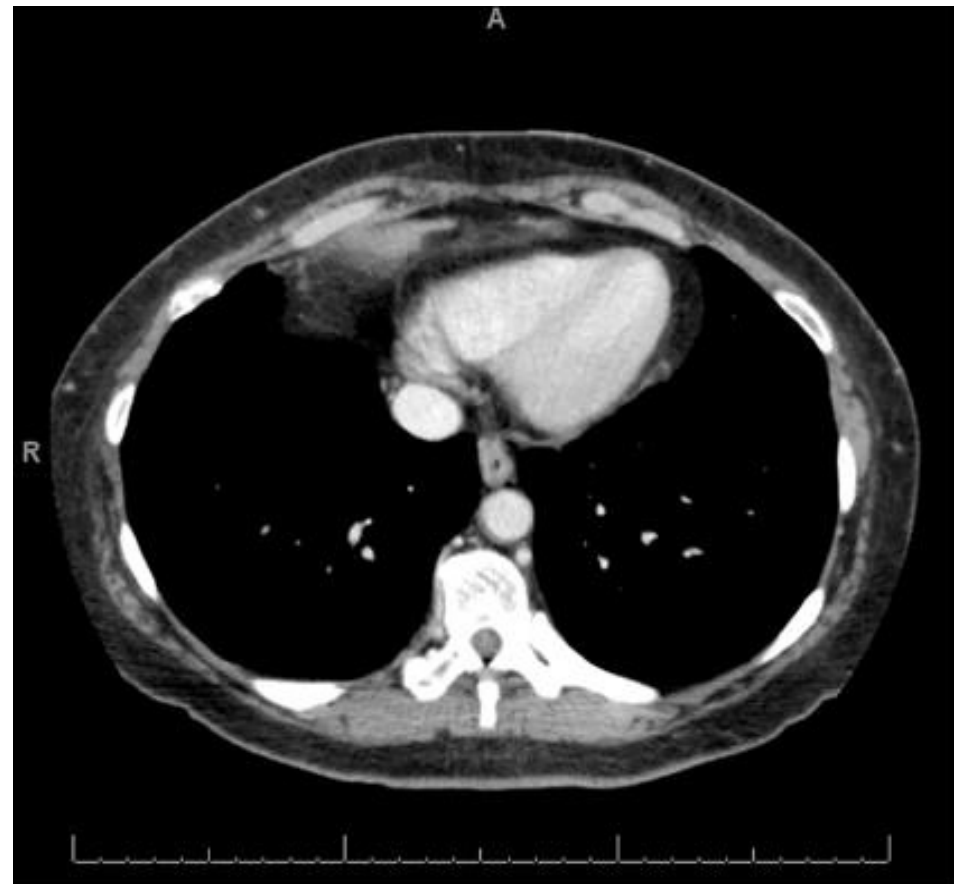

Figure 1. Axial computed tomography showing no clear source of emboli.

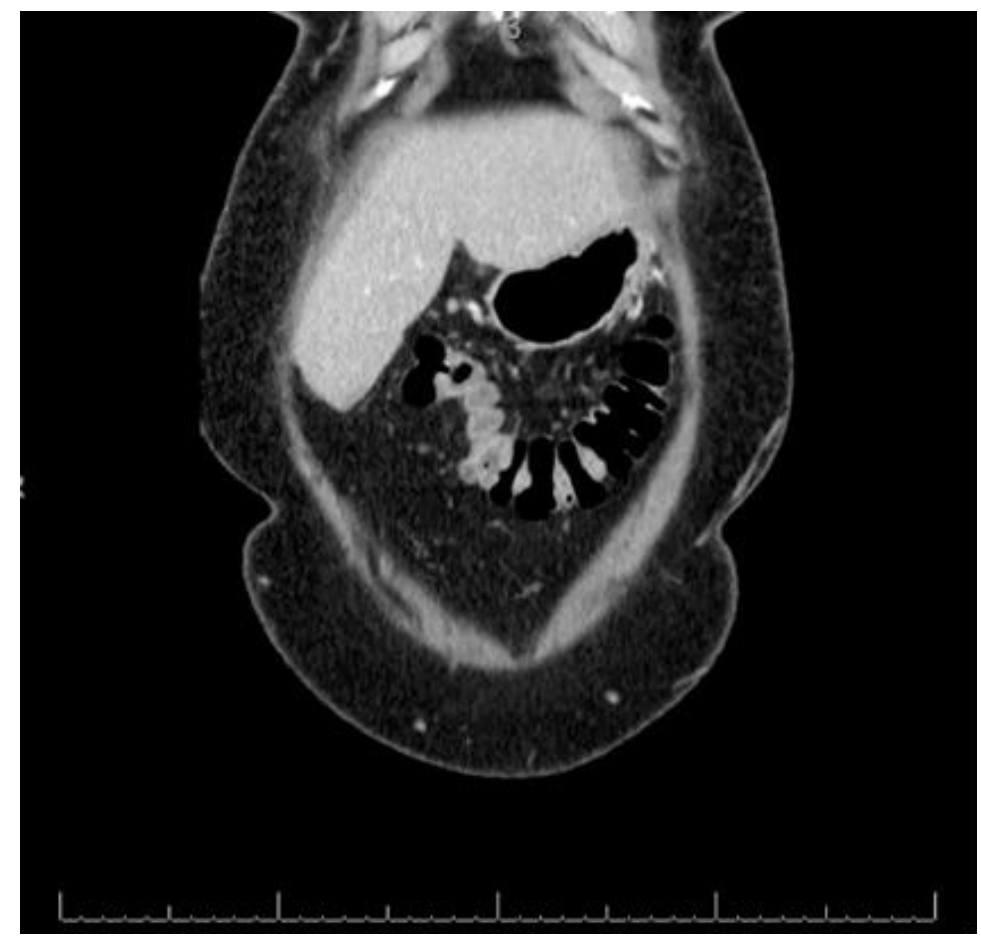

Figure 2. Coronal computed tomography demonstrating a wedge-shaped area of hypoenhancement in the spleen in keeping with a splenic infarct. 
A 52-year-old Hispanic woman with a past medical history significant for Type 1 diabetes mellitus, hypertension, and rheumatoid arthritis presented with left upper quadrant pain for one day. Her review of systems was positive for bloating, severe epigastric and left upper quadrant tenderness that radiated to the back and left shoulder, nausea with non-bilious emesis, and diarrhea for one day prior to admission. Physical exam only revealed epigastric and left upper quadrant tenderness to light palpation without rebound or guarding. Abdominal computed tomography of the abdomen demonstrated a new acute or subacute splenic infarct with no clear evidence of an embolic source in the abdomen or pelvis (Figures 1 and 2). Echocardiogram with bubble study and contrast did not demonstrate valve abnormalities, cardiac mass, vegetation, valve or wall motion abnormalities and no evidence of patent foramen ovale.

Splenic infarction should be suspected when patients present with sharp, acute left upper quadrant pain radiating to the left shoulder (Kehr sign). Splenic friction rub may be auscultated but CT is diagnostic (1). Splenic infarction is an uncommon condition, with compiled reports from a 10-year retrospective study of an academic center showing only 32 cases ( $0.016 \%$ of admission). Causes included cardiogenic emboli (62.5\%), autoimmune disease (12.5\%), associated infection (12.5\%), or hematological malignancy (6\%) (2). Active malignancy, embolic events from atrial fibrillation or infective endocarditis, and inflammatory disorders appear to be consistent causes for splenic infarct as demonstrated in a compilation study of 123 case reports of nontraumatic splenic infarction (3). Management includes supportive care and treating the underlying cause, embolic (e.g. endocarditis), vasculitis, or in situ occlusion from predisposing conditions (e.g. sickle cell, polycythemia vera, chronic myeloid leukemia, or myelofibrosis) (1).

Given her stable condition and improving clinical course, she was discharged to follow up with her outpatient provider with recommendation for an anti-phospholipid and hypercoagulability workup.

Daniel J Casey, MSIV. Faraz Jaffer, MD. and Don Leo Pepito, MD. University of Arizona College of Medicine at South Campus Tucson, Arizona USA

\section{References}

1. LeBlond R, Brown D, DeGowin R. The abdomen, perineum, anus, and rectosigmoid. In: Degowin's Diagnostic Examination. New York, NY: McGraw Hill Medical; 2009: 445-527.

2. Schattner A, Adi M, Kitroser E, Klepfish A. Acute Splenic Infarction at an Academic General Hospital Over 10 Years: Presentation, Etiology, and Outcome. Medicine (Baltimore). 2015 Sep;94(36):e1363. [CrossRef] [PubMed]

3. Cox M, Li Z, Desai V, Brown L, Deshmukh S, Roth CG, Needleman L. Acute nontraumatic splenic infarctions at a tertiary-care center: causes and predisposing factors in 123 patients. Emerg Radiol. 2016 Apr;23(2):155-60. [CrossRef] [PubMed] 